\title{
Transition of Pi2 ULF wave polarization structure from the ionosphere to the ground
}

\author{
P. V. Ponomarenko ${ }^{1,2}$ and and C. L. Waters ${ }^{2}$ \\ Received February 2013; accepted 19 February 2013; published 29 April 2013.
}

[1] Trains of ultra low frequency (ULF) waves in the Pi2 range (40-150 s) are regularly detected in near-Earth space. While some Pi2 occur just before substorm onset, others do not show any casual relation to substorm activity so that the $\mathrm{Pi} 2$ generation and propagation mechanisms are still unclear. The bulk of information on Pi2 morphology is provided by ground-based magnetometers, subject to distortions due to transition through the highly conducting lower ionosphere. While space-borne magnetometers provide important in situ information, they cannot match the spatio-temporal continuity of ground-based instruments. In this paper, we devise a novel approach using co-located high-frequency ionospheric radars and ground magnetometers for the first direct observation of the Pi2 wave polarization transition between the ionosphere and the ground. Furthermore, application of the Hilbert transform enables us to reconstruct the transition dynamics, which resolves an apparent disagreement between the Pi2 spectra measured by the two instruments. Citation: Ponomarenko, P. V., and C. L. Waters (2013), Transition of Pi2 ULF wave polarization structure from the ionosphere to the ground, Geophys. Res. Lett., 40, 1474-1478, doi:10.1002/grl.50271.

\section{Introduction}

[2] Ultra low frequency (ULF, $\simeq 1-100 \mathrm{mHz}$ ) variations of the geomagnetic field caused by plasma waves in the terrestrial magnetosphere have been categorized according to their regular (pulsations continuous, $\mathrm{Pc}$ ) or irregular (pulsations irregular, Pi) temporal structure [Jacobs et al., 1964]. Pi2 pulsations appear as damped sinusoidal variations in the $40-150 \mathrm{~s}$ period range, and some of them have been associated with substorm onset [Saito, 1969; Olson, 1999]. However, identical variations that are not related to substorms have also been reported [Liou et al., 2000]. A recent review by Keiling and Takahashi [2011] summarized the current understanding of $\mathrm{Pi} 2$ generation and propagation in the context of seven models and analyzed the associated experimental evidence. While combined ground/satellite studies have distilled possible generation/propagation scenarios [e.g., Han et al., 2004], they

\footnotetext{
${ }^{1}$ Institute of Space and Atmospheric Studies, University of Saskatchewan, Saskatoon, Saskatchewan, Canada.

${ }^{2}$ School of Mathematical and Physical Sciences, The University of Newcastle, Callaghan, New South Wales, Australia.

Corresponding author: P. V. Ponomarenko, Institute of Space and Atmospheric Studies, 116 Science Place, Saskatoon, SK, S7N 5E2, Canada. (pasha.ponomarenko@usask.ca)

(C)2013. American Geophysical Union. All Rights Reserved. 0094-8276/13/10.1002/grl.50271
}

have not covered effects from the highly conducting lower ionosphere. The ionosphere alters ULF wave properties by (1) acting as a spatial wave number filter [Hughes and Southwood, 1976; Ponomarenko et al., 2001], (2) modifying the amplitude depending on the spatial and temporal scales of the disturbance [Hughes and Southwood, 1976; Yoshikawa et al., 2002; Sciffer et al., 2004] and (3) rotating the polarization ellipse [Nishida, 1964; Hughes, 1974; Southwood and Hughes, 1983; Sciffer et al., 2005]. Therefore, comparison of spacecraft data with ground magnetometer records requires careful consideration of $\mathrm{Pi} 2$ propagation through the ionosphere.

[3] Information about ionospheric ULF wave signatures can be provided by HF/VHF radars. For example, Gjerloev et al., [2007] and Frissell et al., [2011] reported observations of ionospheric Pi2 signatures in the Doppler velocity variations from the Wallops Island HF radar. However, in both of these studies, the ionospheric data were limited to a single line-of-sight velocity component and, therefore, lacked important information on wave polarization. Vertical incidence HF Doppler sounders [e.g., Ikeda et al., 2010] have similar problems because, in this case, Doppler shift is determined by an integral along the propagation path. Here, we make further progress by presenting (1) the first measurements of ULF wave polarization at ionospheric heights using crossed beams from a pair of HF radars and (2) the first direct observation of the ionosphereground transfer of a $\mathrm{Pi} 2$ wave polarization structure using co-located radar and magnetometer data. In addition, application of the Hilbert transform reveals the dynamics of the Pi2 evolution.

\section{Experimental Setup}

[4] The ionospheric properties of $\mathrm{Pi} 2$ waves were obtained through Doppler velocity measurements by two high-frequency (HF, 9-18 MHz) radars from the Super Dual Auroral Radar Network (SuperDARN) [Greenwald et al., 1985; Ponomarenko and Waters, 2006; Chisham et al., 2007]. SuperDARN radars measure Doppler shifts of HF signals backscattered by small-scale $(\sim 10-15 \mathrm{~m})$ ionospheric irregularities at F-region heights $(\sim 300 \mathrm{~km})$. These shifts are generally caused by $\mathbf{E} \times \mathbf{B}_{0}$ plasma drifts, where $\mathbf{B}_{0}$ is the background magnetic field and $\mathbf{E}$ represents ionospheric electric fields of various origins. At high latitudes, the near-vertical orientation of $\mathbf{B}_{0}$ leads to near-horizontal drifts. ULF waves passing through the ionosphere possess an electric field component $\sim 0.1-1 \mathrm{mV} / \mathrm{m}$ that generates quasi-periodic variations in the drift velocity $\sim 10-100 \mathrm{~m} / \mathrm{s}$, which are regularly observed by SuperDARN radars [Ponomarenko et al., 2003]. These radars normally operate with a 1 min scan period when all 16 beams are consecutively sampled using an integration time 


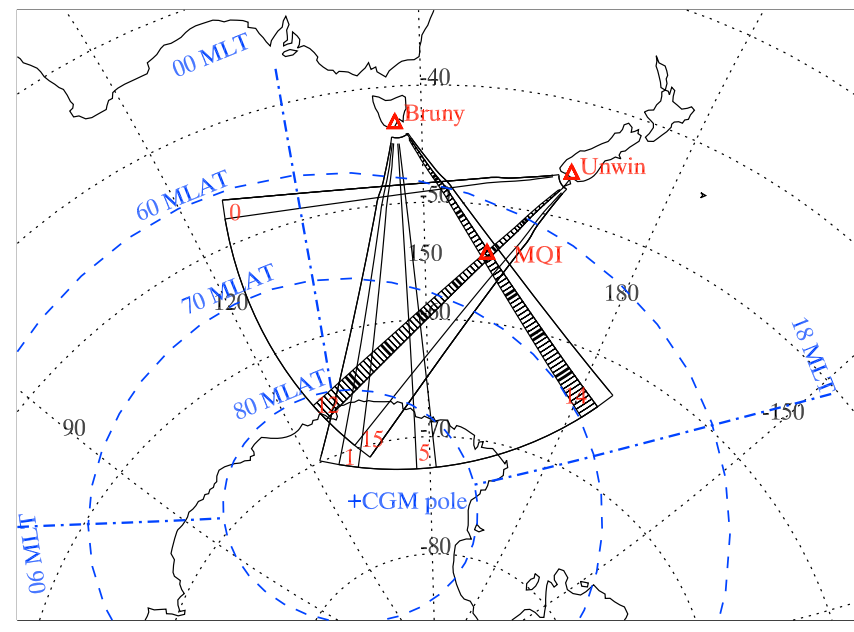

Figure 1. Radar beam configuration for the ULF scan mode. Range gates along each beam are separated by $45 \mathrm{~km}$ (mesh). Thick lines mark every 10th gate starting from gate 0 . The red triangles mark the locations of the Macquarie Island (MQI) magnetometer site and the TIGER Bruny and Unwin radars.

of $\simeq 3 \mathrm{~s}$ per beam (Nyquist frequency $F_{N}=8.3 \mathrm{mHz}$ ). This regime is not particularly suitable for detecting $\mathrm{Pi} 2$ waves (6.67-25 mHz). Therefore, a special "ULF" radar scan mode was designed and implemented in which the radar sampling rate was increased to $\simeq 9 \mathrm{~s}\left(F_{N}=55.5 \mathrm{mHz}\right)$ by reducing the number of sampled beams to three, providing a satisfactory trade-off between $F_{N}$ and spatial coverage. A single SuperDARN radar is only capable of measuring a single line-of-sight (LOS) component of the ionospheric drift velocity along each beam. In order to observe 2D ULF wave polarization properties in the ionosphere, it is necessary to use data from a common ionospheric volume observed simultaneously by two spatially separated radars. To achieve this, data from the pair of Tasman International Geospace Environment Radars (TIGER) radars with overlapping fields-of-view were combined. The location and beam patterns of the TIGER Bruny (Tasmania) and TIGER Unwin (New Zealand) radars are shown in Figure 1. Overlap with the magnetometer site on Macquarie Island (MQI, CGM $64^{\circ} 18^{\prime} \mathrm{S}, 248^{\circ} 21^{\prime} \mathrm{E}, \mathrm{MLT} \simeq \mathrm{UT}+12$ ) was achieved by rotation of the field-of-view of the Bruny radar by $\simeq 7^{\circ}$ counter-clockwise using a specially designed phasing matrix (M. Parkinson, private communication). As a result, beam 14 from Bruny and beam 12 from Unwin intersected above MQI so that the ionospheric ULF wave characteristics could be directly compared with their ground magnetic signatures. The remaining beams ( 1 and 5 for Bruny and 0 and 15 for Unwin) provided supplementary information on the spatial extent of the observed ULF waves.

[5] The magnetic field vector data at ground level were sampled at a 1 s rate using fluxgate magnetometers operated by Geoscience Australia (Commonwealth of Australia (Geoscience Australia) 2012. This product is released under the Creative Commons Attribution 3.0 Australia Licence). The magnetic data components were aligned with magnetic (AACGM) coordinates, so that the North and East directions correspond to positive deviations. For ease of comparison, the radar velocity vectors were converted to the same coordinate system, under the assumption that the LOS vectors for both radars are parallel to the ground.

\section{Pi2 Data for 19 October 2006}

[6] The data recorded over the $1 \mathrm{~h}$ interval preceding a moderate substorm observed on 19 October 2006 were analyzed. The top panel in Figure 2 shows the NS component of the geomagnetic field at MQI, which exhibits a persistent negative excursion starting at $\simeq 10$ UT (22 MLT) and lasting for several hours. The second panel of Figure 2 shows both horizontal components from the same data set, but zoomed to 09-10 UT (21-22 MLT) and with a $300 \mathrm{~s}$ boxcar moving average subtracted. The detrended data reveal several trains of Pi2-like oscillations. Similar variations in the LOS velocity with amplitudes reaching $100 \mathrm{~m} / \mathrm{s}$ were also observed by both radars across range gates 10-30 (group range $900-1500 \mathrm{~km}$ ), which is close to the average loca-
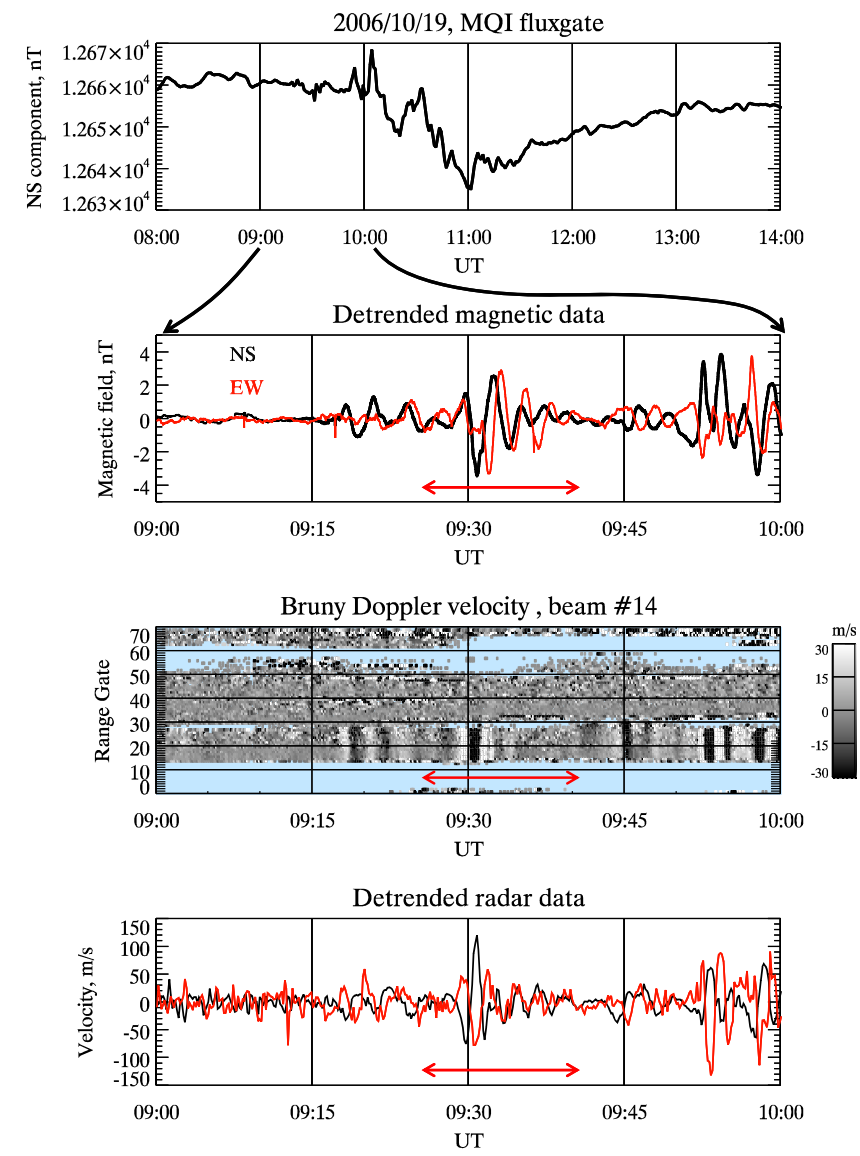

Figure 2. (Top panel) NS component of the geomagnetic field at MQI over 08-14 UT on 19 October 2006. A substorm onset is apparently near 10 UT. (Second panel) NS (black) and EW (red) magnetic field components at MQI zoomed to 09:00-10:00 UT and detrended by subtracting a $300 \mathrm{~s}$ boxcar moving average. Several isolated Pi2 trains are evident between 09:15 UT and the substorm onset. (Third panel) UT-range plot of Bruny beam 14 Doppler velocity data (09:00-10:00 UT, detrended). (Bottom panel) similar to the second panel but for the radar velocity data. Red arrows mark the 9:25-9:40 UT event analyzed in detail in this paper. 
tion of the nightside plasmapause. To illustrate the radar signatures, the third panel of Figure 2 shows velocity data for beam 14 of the Bruny radar. Following Ponomarenko et al., [2003], these data were preprocessed by subtracting a $300 \mathrm{~s}$ moving average to remove the slow-varying ionosphere drifts. The velocity variations are plotted in saturated gray scale, while blue shading denotes missing data. The radar $\mathrm{Pi} 2$ signatures appear as vertical stripes of alternating shades of grey depicting synchronous velocity oscillations across a number of range gates. The bottom panel shows NS (black) and EW (red) components of the ionospheric drift velocity over MQI reconstructed from the crossedbeam radar data. The radar range cells located over MQI are gate 30 for Bruny and gate 22 for Unwin (Figure 1). While gate 22 on beam 12 of Unwin contains continuous data during the interval of interest, there is a small data gap in the Bruny data over gates 28-32. This was filled using the closest available gate containing valid data, gate 27 , based on the high coherency of the velocity variations across at least 15 range gates. The selected radar records also contain a few outliers, which were identified and replaced by linear interpolation between neighboring valid data. Results from $\mathrm{HF}$ ray tracing for the given ionospheric conditions favor the interpretation of these echoes as 1/2-hop ionospheric backscatter. Similar Pi2 wave trains were observed nearly simultaneously across the whole $210^{\circ}$ meridian magnetometer array (not shown) indicating the broad latitudinal extent of these events. For illustrative purposes, here, we focus on a pronounced ULF signature observed over 09:2509:40 UT (21:25-21:40 MLT) as marked by red arrows in Figure 2. This event exhibited all the characteristic features of $\mathrm{Pi} 2$ waves, but occurred well before the substorm onset at $\simeq 10 \mathrm{UT}$, so it does not represent a conventional precursor.

[7] Fourier analysis, supplemented by Hanning windowing, was applied to the unfiltered time series covering the 09:25-09:40 UT interval. Figure 3a shows normalized trace power (sum of spectral powers of the orthogonal components) for the radar (red) and magnetometer (black) data sets. The dominant peaks are located around 7-8 $\mathrm{mHz}$, while significant spectral power is limited to frequencies below $\simeq 20 \mathrm{mHz}$. In order to analyze ULF wave polarization, the equations for ellipticity and azimuth, based on Stokes parameters as described by, e.g., Fowler et al. [1967], were used. The respective spectra are plotted in Figures $3 \mathrm{~b}$ and $3 \mathrm{c}$. For the coordinates used, positive values of azimuth correspond to the angle measured clockwise from the magnetic North, and positive ellipticity values (sense of polarization) describe clockwise rotation of the polarization ellipse. Figure $3 d$ represents the amplitude transition coefficient "ionosphere-ground" measured in $\mathrm{nT} /(\mathrm{m} / \mathrm{s})$. The black vertical dashed lines in Figure 3a indicate the $3-30 \mathrm{mHz}$ band used in complex decomposition analysis (see below).

[8] The Pi2 pulsations represent wave packets, which are confined in time. Therefore, important information on their temporal evolution can be missed when using conventional Fourier analysis. In order to observe the time history of the event, the complex decomposition technique was applied. This conventionally provides phases and amplitudes of the signal at the dominant spectral component sampled at the same rate as the signal itself. The major limitation of this technique is that it is applicable to narrow-band signals, and Figure $3 \mathrm{a}$ indicates that this requirement is satisfied. In order to accommodate the $\mathrm{Pi} 2$ frequency range $(6.67-25 \mathrm{mHz})$ and minimize contamination by the lowerand higher-frequency components, all data sets were bandpass-filtered to suppress spectral power outside the 3-30 $\mathrm{mHz}$ band. The complex signal, $S(t)$, was constructed using the recorded signal $s(t)$ as the real part and its Hilbert transform, $H(s(t))$, as the imaginary part, i.e., $S(t)=s(t)-$ $i H(s(t))=|S(t)| e^{i \phi}$, where $i=\sqrt{-1}$ and $|S(t)|$ and $\phi(t)$ are the time dependent amplitude and phase of the complex signal, respectively.

[9] In order to obtain time dependent polarization characteristics, the complex signals were processed using the same expressions from [Fowler et al., 1967], and the results are shown in the respective right-hand panels of Figure 3. For ease of comparison, the magnetic data, which were originally sampled at $1 \mathrm{~s}$, are shown only at the times coinciding with the radar samples, i.e., every 9 s.

[10] To ensure that the polarization properties are calculated for the same periodicities, it was important to examine the temporal history of the signal frequency for both data sets. This was achieved in two steps. First, instantaneous phases for both orthogonal components, $\phi_{x}$ and $\phi_{y}$, were differentiated in the time domain to obtain the respective frequencies, $f_{x, y}(t)=(1 / 2 \pi)\left(\partial \phi_{x, y}(t) / \partial t\right)$. Second, the effective frequency of the polarized wave was calculated as a weighted average, $f=\left(f_{x} p_{x}+f_{y} p_{y}\right) /\left(p_{x}+p_{y}\right)$, where the weighting was based on the respective powers, $p_{x}$ and $p_{y}$. This parameter is plotted in Figure $3 \mathrm{~h}$ by black (ground) and red (ionosphere) diamonds using the right-hand $Y$ axis.

\section{Experimental Summary and Discussion}

[11] The polarization spectra computed from the conventional Fourier analysis in Figures $3 \mathrm{a}-3 \mathrm{~d}$ show a number of differences between the ionospheric and ground Pi2 signatures. While the trace power spectra overlap quite well in the frequency domain, they possess slightly different maximum frequencies, $\simeq 7 \mathrm{mHz}$ on the ground and $\simeq 8 \mathrm{mHz}$ in the ionosphere. More importantly, there is little resemblance in the respective ellipticity and azimuth spectra. There are two groups of factors that might lead to this: physical and instrumental.

[12] With respect to physical factors, one should expect some changes in the wave's characteristics resulting from its transition between the ionosphere and the ground. The slight shift in the maximum frequency (Figure 3a) could be related to the screening of higher frequency/smaller scales, and this interpretation seems to be supported by the decrease of the transfer function with increasing frequency (Figure 3d). With respect to azimuth, early works [e.g., Southwood and Hughes, 1983] predicted a $90^{\circ}$ rotation of the polarization ellipse on the ground with respect to its orientation above the ionosphere. If we assume that the measured radar velocity variations are caused by $\mathbf{E} \times \mathbf{B}$ drift of the fully magnetized plasma, then the wave magnetic field oscillations are aligned with those in the plasma drift. This means that the observed azimuth change (Figure $3 \mathrm{c}$ ) is smaller than expected in magnitude and rather complex in nature. The reduced amount of rotation can be explained by more realistic models, which account for a non-vertical geomagnetic field, wave mode conversion coefficients and horizontal nonuniformity of the E-layer conductivity. For example, Sciffer 

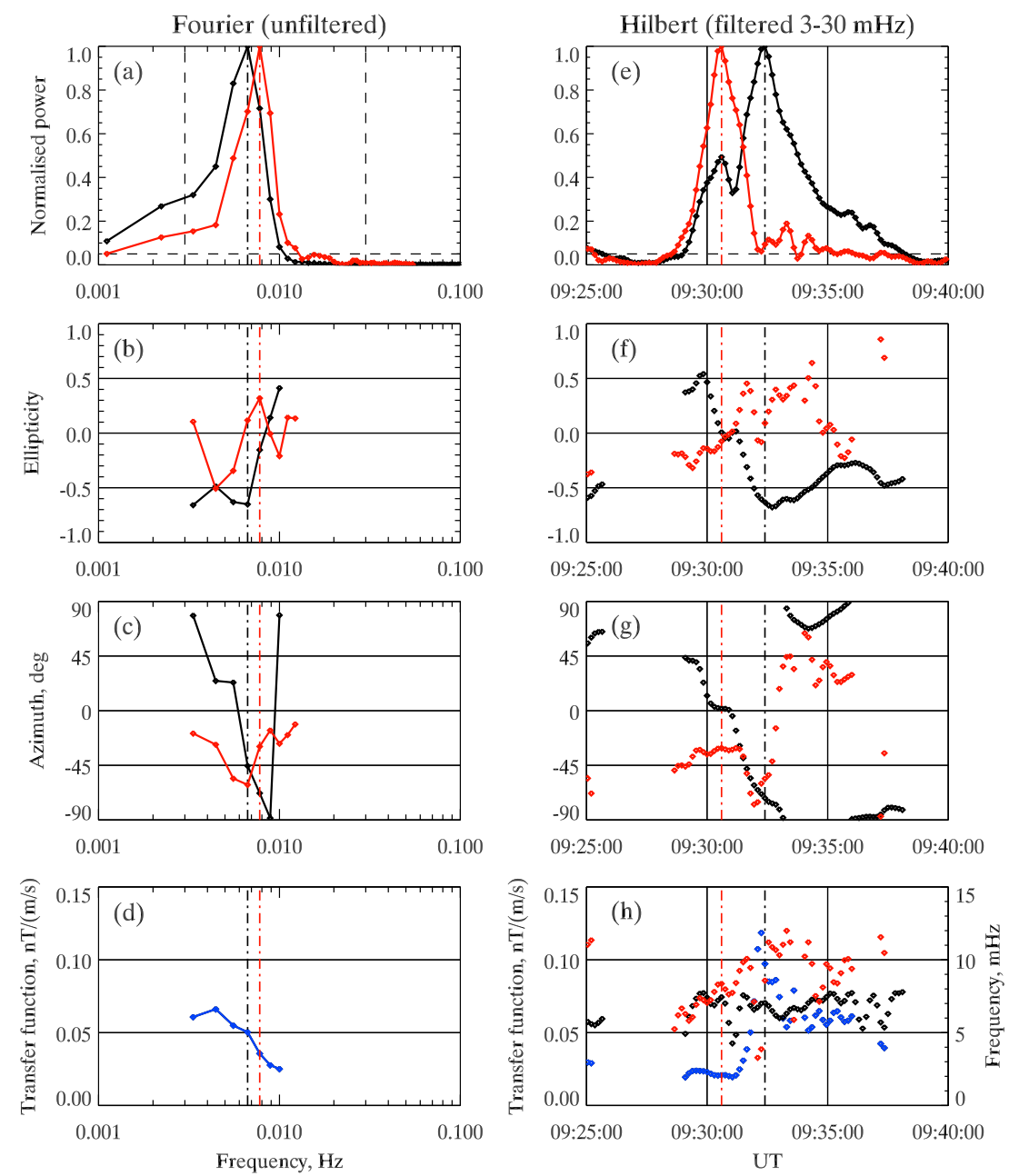

Figure 3. Comparison of polarization characteristics of the radar (red) and magnetometer (black) data for 9:25-9:40 UT, 19 October 2006, obtained using (a-d) the Fourier transform and $(\mathrm{e}-\mathrm{f})$ the complex decomposition based on the Hilbert transform. The vertical dash-dot lines correspond to the maximum power in the respective data sets. The vertical dashed lines in Figure 3a show the frequency band used for the complex decomposition analysis (right column). The horizontal dashed lines in Figures 3a and $3 \mathrm{f}$ indicate a 5\% power cutoff used for plotting the polarization characteristics in the lower panels. The black and red diamonds in Figure $3 \mathrm{~h}$ correspond to the right $Y$ axis and represent instant frequencies, while the blue symbols depict the transfer function (left $Y$ axis, see text for more details).

et al. [2005] showed that the azimuth rotation depends on the relative contributions from fast and shear Alfvén modes in the topside ionosphere, so it is altitude dependent. However, our spectral data also show opposite rotation signs at the ionospheric and ground spectral maxima, while the respective ellipticity values exhibit opposite signs and differing magnitudes. While these observational details appear to be unexplainable within our current understanding of the ULF wave transfer through the ionosphere, they can be reconciled with theory by analyzing the temporal evolution of the ULF wave.

[13] At first glance, the instantaneous Pi2 characteristics in Figures $3 \mathrm{e}-3 \mathrm{~h}$ also show little resemblance between the ionosphere and the ground. The trace power in the ionosphere shows a single peak at 09:30:30 UT, while the ground power peaks at $\simeq 9: 32: 30$ UT showing only a secondary enhancement coinciding with the ionospheric maximum. The respective ellipticity and azimuth time series also change in apparently independent ways. However, a closer look at the polarization characteristics near the coincident peaks at $\simeq 9: 30: 30$ UT indicates that these peaks have a common origin. These indicators include (1) nearly linear polarizations (Figure 3f), (2) consistent azimuth shift of $\simeq 40^{\circ}$ (Figure $3 \mathrm{~g}$ ), (3) constant transfer function at $\simeq 0.025 \mathrm{nT} /(\mathrm{m} / \mathrm{s})$ (Figure 3h, blue), and (4) close instantaneous frequencies at $\simeq 7.5 \mathrm{mHz}$ (red and black diamonds in Figure $3 \mathrm{~h}$ ).

[14] The existence of the second (major) temporal power peak in the ground record might be related to instrumental effects arising from the different spatial coverage of the diagnostic tools used to analyze ULF wave signatures in the ionosphere and on the ground. Above MQI, the radars provide a significantly better spatial resolution determined by the $45 \mathrm{~km}$ range gate cell along LOS and $50-100 \mathrm{~km}$ across it. In contrast, the ground magnetometers integrate the signal from a much larger area of the ionosphere. Therefore, the second ground maximum may originate from an active ionospheric region, which lies away from the zenith 
direction at MQI, so it is unseen by the radar, but makes a major contribution to the ground data. As a result, the spectral characteristics of the ionospheric and ground data may be dominated by different spatio-temporal components, thus explaining the observed differences in the Fourier spectra. The currently available information is insufficient to clarify the nature of this additional component. Finally, assuming that the observed $\mathrm{Pi} 2$ event preserves its linear polarization over the field-of-view of the radars, we estimated the azimuthal wave number using data from additional beams of the Bruny radar, $m \simeq-(5-6)$ with the minus sign indicating westward phase propagation.

\section{Conclusions}

[15] In this paper, we have reported the first direct observation of the ionosphere-ground transition of the polarization characteristics for a Pi2 wave in the vicinity of the nightside plasmapause using Doppler velocity records from a pair of SuperDARN HF radars and co-located ground magnetometer data. Application of the Hilbert transform reveals the temporal evolution of the event and resolves the discrepancy between the average pulsation characteristics in the ionosphere and on the ground, which apparently arises from the difference in the spatial resolution of the instruments.

[16] For this particular event, the approximate linear polarization of the $\mathrm{Pi} 2$ wave was transferred from the ionosphere to the ground, accompanied by an $\simeq 40^{\circ}$ rotation of the polarization ellipse. The amplitude transfer ratio was nearly constant at $0.025 \mathrm{nT} /(\mathrm{m} / \mathrm{s})$, and the azimuthal wave number was $\simeq 5-6$ westward. This information is vital for understanding the ionosphere-ground transition of Pi2 waves and how spacecraft measurements are related to ground magnetometer signatures of Pi2 signals.

[17] Acknowledgement. This work was supported by the Australian Research Council, the University of Newcastle, the University of Saskatchewan, and operation funds from Canadian Research Chair J.-P. St.-Maurice.

\section{References}

Chisham, G., et al. (2007), A decade of the Super Dual Auroral Radar Network (SuperDARN): Scientific achievements, new techniques and future directions, Surv. Geophys., 28, 33-109, doi:10.1007/ s10,712-007-9017-8.
Fowler, R. A., B. J. Kotick, and R. D. Elliott (1967), Polarization analysis of natural and artificially induced geomagnetic micropulsations, J. Geophys. Res., 72(11), 2871-2883, doi:10.1029/JZ072i011p02871.

Frissell, N. A., et al. (2011), First radar observations in the vicinity of the plasmapause of pulsed ionospheric flows generated by bursty bulk flows, Geophys. Res. Lett., 38, 2790, doi:10.1029/2010GL045857.

Gjerloev, J. W., et al. (2007), Observations of Pi2 pulsations by the Wallops HF radar in association with substorm expansion, Geophys. Res. Lett., 34 (L20103), doi:10.1029/2007GL030492.

Greenwald, R. A., et al. (1985), An HF phased-array radar for studying small-scale structure in the high-latitude ionosphere, Radio Sci., 20, 63-79.

Han, D. S., et al. (2004), A comparative analysis of low-latitude Pi2 pulsations observed by Oersted and ground stations, J. Geophys. Res., 109 (A10209), doi:10.1029/2004JA010576.

Hughes, W. J. (1974), The effect of the atmosphere and ionosphere on long period magnetospheric micropulsations, Planet. Space Sci., 22, $1157-1172$.

Hughes, W. J., and D. Southwood (1976), The screening of micropulsation signals by the atmosphere and ionosphere, J. Geophys. Res., 81, 3234-3240.

Ikeda, A., et al. (2010), Phase relation between Pi2-associated ionospheric Doppler velocity and magnetic pulsations observed at a midlatitude MAGDAS station, J. Geophys. Res., 115 (A02215), doi: 0.1029/2009JA014397.

Jacobs, J. A., Y. Kato, S. Matsushita, and V. A. Troitskaya (1964), Classification of geomagnetic pulsations, J. Geophys. Res., 69, 180-181.

Keiling, A., and K. Takahashi (2011), Review of Pi2 models, Space Sci. Rev., 161, 63-148, doi:10.1007/s11,214-011-9818-4.

Liou, K., et al. (2000), Evaluation of low-latitude Pi2 pulsations as indicators of substorm onset using polar ultraviolet imagery, J. Geophys. Res., $105,2495-2505$.

Nishida, A. (1964), Ionospheric screening effect and storm sudden commencement, J. Geophys. Res., 69, 1861-1874.

Olson, J. V. (1999), Pi2 pulsations and substorm onsets: A review, J. Geophys. Res., 104, 17,499-17,520.

Ponomarenko, P. V., and C. L. Waters (2006), Spectral width of SuperDARN echoes: Measurement, use and physical interpretation, Annales Geophysicae, 24, 115-128, www.ann--geophys.net/25/675/2007/.

Ponomarenko, P. V. et al. (2001), Spatial structure of ULF waves: Comparison of magnetometer and super dual auroral radar network data, $J$. Geophys. Res., 106, 10,509-10,517.

Ponomarenko, P. V., F. W. Menk, and C. L. Waters (2003), Visualization of ULF waves in SuperDARN data, Geophys. Res. Lett., 30, 1926-1929, doi:10.1029/2003GL017757.

Saito, T. (1969), Geomagnetic pulsations, Space Sci. Rev., 10, 319-412.

Sciffer, M. D., C. L. Waters, and F. W. Menk (2004), Propagation of ULF waves through the ionosphere: Inductive effect for oblique magnetic fields, Ann. Geophy., 22, 1155-1169, doi:10.5194/angeo-22-1155-2004.

Sciffer, M. D., C. L. Waters, and F. W. Menk (2005), A numerical model to investigate the polarisation azimuth of ULF waves through the ionosphere with oblique magnetic fields, Ann. Geophys., 23, 3457-3471, doi: 10.5149/angeo-23-3457-2005.

Southwood, D. J., and W. J. Hughes (1983), Theory of hydromagnmetic waves in the magnetosphere, Space Sci. Rev., 35, 301-366.

Yoshikawa, A., et al. (2002), Hall-induced inductive shielding effect on geomagnetic pulsations, Geophys. Res. Lett., 29, doi:10.1029/ 2001GL013,610 